\title{
LA BUROCRACIA COMO ÉLITE: \\ EL ESLABÓN PERDIDO EN LOS ESTUDIOS SOBRE ELITES \\ GUBERNAMENTALES EN CHILE
}

\author{
Juan Pablo Araya Orellana \\ Universidad de Chile \\ jpabloaraya@iap.uchile.cl
}

\begin{abstract}
RESUMEN
El presente estudio tiene como propósito aportar evidencia para la conceptualización de la burocracia pública como élite política en Chile. A través del análisis del status político y los roles políticos, esta investigación explora en los mecanismos utilizados por los burócratas de nivel medioalto mediante los cuáles ejercen influencia en la formulación de política pública. Este artículo analiza el caso de la política de modernización de la gestión pública en Chile (1990 - 2014). A través del análisis de entrevistas de setenta y ocho diferentes actores de esta política, es posible reconocer la influencia de los burócratas de nivel medio a través de un tipo especial de experticia (experticia de política pública), explicada por la estructura de carrera, el alto grado de politización de la burocracia chilena y el tipo de trabajo realizado, lo que formaría una especie de capital especial de los burócratas: el capital burocrático.
\end{abstract}

Palabras clave: Élites, Burocracia, Burócratas de nivel medio, Tecnocracia, Chile. 


\title{
BUREAUCRACY AS AN ELITE: \\ THE MISSING LINK IN STUDIES ON GOVERNMENTAL ELITES IN CHILE
}

\begin{abstract}
The aim of this study is to contribute to the construction of a theoretical framework for the subject of public bureaucracy as a political elite in Chile. Through an analysis of their political status and political roles, this research explores the mechanisms used by medium-high level bureaucrats to exert influence in the formulation of public policies. This article analyzes the public management modernization policy (1990-2014). The analysis of interviews with 78 different actors of this policy makes it possible to see the influence middle-level bureaucrats have thanks to their special type of expertise (public policy expertise) that has to do with the career structure, the high level of politicization of Chilean bureaucracy and the type of tasks that are carried out, which would conform a sort of special capital of bureaucrats: the bureaucratic capital.
\end{abstract}

Keywords: Elites, Bureaucracy, Middle-level bureaucrats, Technocracy, Chile. 


\section{INTRODUCCIÓN}

En las últimas décadas es posible constatar un creciente interés desde la sociología y la ciencia política, principalmente, con respecto al estudio de élites en Chile. Una gran parte de estos estudios abordan las élites desde una perspectiva histórica, a través del estudio biográfico de personajes importantes (Stuven 2000, Stabili 2003, Vicuña 1996, Vicuña 2001). Según Joignant (2009), las recientes investigaciones sobre élite en Chile se han centrado más bien en el análisis de agentes gubernamentales y de sus capitales, los cuales les permiten influir en las decisiones políticas. Dentro de estos agentes, Joignant (2009) centra su estudio en el análisis de los economistas y su influencia en altas posiciones gubernamentales, en virtud de su alta formación intelectual. Estos agentes habrían transitado desde posiciones asesoras, las que históricamente habrían ocupado, hacia roles centrales en la dirección del gobierno (Silva 2008, Hunneus 1998, Montecinos y Markoff 2009).

Siguiendo con esta premisa, pero ampliando el foco al análisis a otros grupos profesionales, un grupo de investigaciones evidencia la influencia de los tecnócratas y tecnopolíticos en Chile (Dávila 2011, Joignant 2011, Mella 2008, Silva 2008, Joignant y Güell 2011, Delamaza 2011). La mayoría de estos estudios ha analizado las trayectorias de agentes políticos en los gabinetes ministeriales, específicamente la figura de ministros y subsecretarios. Los tecnócratas son actores con una alta experticia técnica (la que, generalmente, es evidenciada por la posesión grados de doctorado en universidades de prestigio estadounidenses o europeas), con alta formación académica, ejerciendo principalmente una función asesora. Por otro lado, los tecnopolíticos, aunque comparten con el tecnócrata la característica de una alta experticia técnica, se diferenciarían por poseer un capital político (Joignant 2011, Domínguez 1997). Silva (2008) realiza un análisis histórico de la influencia de estos actores a lo largo del siglo XX, evidenciando durante el período comprendido entre 1990 y el 2010, una marcada influencia de una élite tecnocrática. Por otro lado, desde la vereda del análisis de política pública, Olavarría (2015) compara el proceso de formulación de cuatro políticas públicas en Chile, entre 1990 y 2014, concluyendo que la formulación de política en Chile se caracterizaría por la presencia de la siguiente estructura: un tecnopolítico empoderando a un grupo de tecnócratas, con el objetivo de diseñar e implementar

1 Las políticas públicas analizadas por Olavarría (2015) son: la política de modernización de la gestión pública, el plan de transporte público de Santiago (Transantiago), la reforma de salud y el Plan Auge, Ley de Transparencia y Acceso a la Información Pública. 
determinadas políticas públicas.

Finalmente, un tercer grupo de investigaciones ha estudiado a los políticos profesionales (en términos weberianos) como élite. Según Delamaza (2013), los rasgos característicos de la élite política chilena postdictatorial se asocia con la presencia del elitismo democrático y la expansión de la tecnopolítica. En particular, con respecto al primer elemento caracterizador evidenciado por Delamaza (2013), gran parte de la elite política chilena tiene sus orígenes en los movimientos de la sociedad civil pro democracia, principalmente en la década de 1980, siendo estos actores legitimados en sus posiciones por estos mismos procesos eleccionarios democráticos. Como señala Delamaza (2013: 5) “[...] la estabilidad política de los siguientes veinte ańos (desde 1990 en adelante), permitió la conformación progresiva de una poderosa élite".

Sin embargo, con respecto al análisis de la burocracia pública chilena ${ }^{2}$ existen escasas investigaciones que identifiquen el tipo de capital que le permitiría ejercer influencia política. En este punto es necesario precisar que, aunque los tecnócratas y los tecnopolíticos pueden ocupar un mismo campo de acción con los burócratas, son distintos entre sí. Por un lado, los tecnócratas, quienes se ubican en cargos medios-altos, se caracterizan por ejercer influencia producto de una alta experticia técnica, evidenciada principalmente en la posesión de grados de doctorado, y buscarían mediante el método científico y la racionalidad científica soluciones óptimas a los problemas públicos (Günell 1982, Centeno y Maxfield 1992, Centeno 1993, Cheng y White 1990, Collier 1979, Mizuno 2009, Silva 2008, Rowney 1989, Wonka 2007, Ribbhagen 2013). También se caracterizarían por mirar con apatía el juego político que da origen a las decisiones de política pública (Ridley 1966, Baylis 1974, Putnam 1977). Por otro lado, los tecnopolíticos, ubicados en posiciones altas del Estado, si bien influyen por su alta experticia técnica, evidenciada principalmente en la posesión de grados doctorales, poseen un importante capital político, casi siempre manifestado por la pertenencia a un partido o movimiento político, lo que también les da influencia política en la formulación de política pública (Williamson 1994, Domínguez 1997; en relación con el capital político de los tecnopolíticos, Joignant 2011).

A diferencia de los tecnócratas y los tecnopolíticos, los burócratas, y en particular los burócratas de nivel medio-alto no influencian en función de su experticia técnica, ya que, generalmente, no poseen grados doctorales

2 Se entiende la burocracia como todos aquellos puestos excluidos de la exclusiva confianza política, de acuerdo al orden jurídico-institucional chileno 
ni trayectorias académicas previas, por lo que actores como los tecnócratas están más capacitados para prescribir soluciones o recomendaciones. Y si bien la literatura señala que los burócratas participarían de ciertas redes políticas, su influencia no se basa en la pertenencia a algún partido o movimiento político. Más bien la influencia de los burócratas de nivel medio-alto estaría determinada por un tipo particular de experticia, más asociada a un conocimiento tácito de las prácticas institucionales de carácter forma o informal. (Alexander et al. 2011, Page y Jenkins 2005, Howlett y Walker 2012, Colebatch, Hoppe y Noordegraaf 2010, Noordegraaf 2015).

Llama la atención las pocas referencias en las ciencias sociales en Chile a los burócratas de nivel medio-alto ${ }^{3}$ como agentes de influencia en las decisiones gubernamentales, más aún cuando la revisión histórica relacionada con la construcción del Estado en Chile destaca el interés de ciertos grupos por formar parte de la burocracia estatal (Góngora 1981, Salazar 2006, Jáksic 2013). Esta omisión de la investigación de la burocracia como élite gubernamental es aún más llamativa, al observar los rasgos del sistema político chileno, con un marcado hiperpresidencialismo, lo que le otorga una influencia importante a los actores al interior del poder ejecutivo (O’Donnell 1992, Shuggart y Mainwaring 1997, Shuggart y Haggard 2001, Samuels y Shugart 2003), entre ellos, los burócratas de nivel medio-alto. Los estudios con respecto a la burocracia chilena a finales del siglo XX analizan tangencialmente la burocracia como espacio de poder, sin embargo el foco principal está puesto en resaltar los rasgos negativos de la influencia política de los burócratas, en parte influido por la corriente del public choice, dejando de lado el estudio de la influencia política de los burócratas en la formulación de las políticas (Rehren 2002, 2004). Por ende, cobra una especial relevancia el estudio de los burócratas chilenos de nivel medio, analizando sus principales caracterísicas como trayectoria o educación, entre otras, y explorar los mecanismos mediante los cuales podrían ejercer una influencia más allá de la implementación o ejecución de programas o políticas, con el objetivo de obtener una imagen inicial de este grupo de agentes gubernamentales.

Es probable que una de las causas de la omisión del estudio de los burócratas como élite pueda ser explicada por la observación normativa sobre la necesidad de una separación entre política y administración, influida por el pensamiento weberiano y wilsoniano (Wilson 1887). Por consiguiente, cualquier evidencia de una influencia política de la

3 A lo largo de esta investigación, cuando hablemos de burócratas, nos estaremos refiriendo a los burócratas de nivel medio-alto, salvo que explícitamente se señale lo contrario 
burocracia es conceptualizada como una anomalía de la dicotomía políticaadministración, y caracterizada como patrimonialismo, generando puntos ciegos en la observación de los burócratas como agentes de influencia.

La presente investigación busca responder a la pregunta sobre si es posible conceptualizar a la burocracia como élite política en Chile. La hipótesis del trabajo es que los burócratas de nivel medio-alto (o burócratas de política pública $\left.{ }^{4}\right)$ presentan rasgos que permitirían identificarla como élite gubernamental en función de su status político y los roles políticos que ejercen. La evidencia empírica fue recolectada en base a entrevistas realizadas a 78 actores involucrados en la política de modernización de la gestión pública en Chile, entre los años 1990 y 2014. Dentro de este grupo, se entrevistó a 30 actores burócratas de nivel medio-alto, quienes tuvieron un rol fundamental en la definición de esta política pública. En una primera parte se abordarán los elementos teóricos.

\section{LA BUROCRACIA COMO ELITE}

Como mencionamos en la introducción, parte importante de la literatura de la ciencia política y política pública ha propuesto una separación entre el campo de acción de los políticos y el de los burócratas. Esta tensión ha sido conceptualizada como la dicotomía política-administración. En distintas versiones, un conjunto de autores ha señalado que el diseño de política estaría dominado por los políticos quienes, definirían los principales objetivos y metas de la política, donde la burocracia no tendría mayor influencia, mientras que en la etapa de implementación, la burocracia se encargaría de traducir las orientaciones de la autoridad en acciones concretas. (Wilson 1887, Goodnow 1900, Simon 1965, Osborne y Gaebler 1992). Aportes más recientes han visto en la dicotomía políticaadministración la condición necesaria para el desarrollo de la neutralidad política de los burócratas (Overeem 2005, 2006).

No obstante, más allá de ser una aproximación teórica prescriptiva, en la realidad es casi imposible observar esta dicotomía. Incluso el mismo Weber (2006), quien pensaba que el modelo ideal sería una separación entre la burocracia y la política, señalaba que en la práctica esta distinción era insostenible. Aberbach et al. (1981) evidencian que, tanto los

4 Esta investigación se centra, específicamente, en los burócratas de nivel medio y alto (jefes de servicio, jefes de división, directivos públicos), grupo que ha sido definido según la literatura como burócratas de política pública (Page y Jenkins 2005, Howlett y Walker 2012, Colebatch 2006, Colebatch, Hoppe y Noordegraaf 2010, Noordegraaf 2015). 
burócratas como los políticos, participan en el proceso de formulación de política pública. En la misma línea de Aberbach et al. (1981), un importante número de investigaciones señala que la burocracia tiene un rol no solamente técnico sino también político, incidiendo directamente en el proceso de formulación de política (Waldo 1952, Merton 1957, Seidman 1970, Allison 1971, Wilson 1989, Peters 1995, Page y Jenkins 2005, Ramesh 2008, Dahlström 2012, Rouban 2012). Lo anterior se basa en una conceptualización más amplia de la política, entendiéndola como la asignación autoritativa de valores (Easton 1965, Lasswell 1936). Por consiguiente, en el marco de esta definición, los burócratas constantemente asignan valores en las decisiones periódicas que toman, por lo que constantemente estarían tomando decisiones políticas. También Svara (2006), caracteriza la relación entre burócratas y políticos como compleja, y aunque reconoce que hay funciones separadas entre administración y política, existe otro conjunto de actividades donde las actividades de la política y la burocracia se superponen o se complementan.

Rouban (2012:382) señala que existe un rango de situaciones “[...] donde las decisiones tomadas por los burócratas reemplazan las decisiones que normalmente fueron tomadas por los gobernantes". Según Savoie (1999), esto dependería de variables contextuales, como por ejemplo la capacidad real del ministro para ejercer liderazgo político sobre los burócratas a su cargo. En un estudio empírico, Page (2012) evidencia diferentes políticas públicas en Reino Unido que fueron definidas en el proceso de redacción de reglamentos (legislación secundaria). En específico señala que

[...] el proceso de redacción no fue una simple traducción del lenguaje de la política y las políticas públicas a los libros de estatutos. Más bien fue, en términos de los tipos de temas en juego y sus efectos sobre lo fue realmente promulgado, una parte crucial del proceso de formulación de política pública (Page 2012: 6).

Carpenter (2001) define esta capacidad burocrática de influir en las decisiones como autonomía burocrática, la cual se produciría por la reputación una determinada agencia y las redes de organizaciones que la respaldan.

En relación con la comprensión de la burocracia como élite, Page y Wright (1999) evidencian dos perspectivas en el análisis: el status político y los roles políticos de los burócratas. Por status político los autores entienden el 
[...] carácter de la élite burocrática y su posición al interior de la maquinaria del Estado [...] las características internas de la alta dirección pública como un grupo social y político, y el rol de los outsiders (los políticos) en darle forma a este grupo" (Page y Wright 1999: 6-7).

Los roles políticos son "[...] las funciones que los burócratas de alto nivel asumen, sea como coordinadores de política pública gubernamental o intermediarios con grupos de interés" (Page y Wright 1999: 6).

Con respecto al análisis del status político de la burocracia, existen ciertos elementos a tener en consideración: 1) el patrón de reclutamiento de los burócratas, el cual puede estar determinado por la formación en instituciones de élite, como grandes écoles de formación de burócratas, o por una alta experticia científica, independiente de la institución donde estos se hayan formado5; 2) la estructura de carrera, en particular de los cargos altos de la burocracia, la que varía en cierto grado en relación al tipo de formación de los burócratas. En aquellos casos donde la formación es más generalista (como el caso de Francia o Inglaterra), los burócratas permanecen en promedio dos ańos en sus cargos, para emigrar a otros cargos de la élite administrativa en otras reparticiones públicas (Mangset 2015a). Por otro lado, en aquellos sistemas donde la formación es más especializada, como en los casos de Noruega (Mangset 2015a, Mangset 2015b), Holanda, Alemania o Italia, los burócratas tienden a desarrollar sus carreras en una misma institución (Page y Wright 1999: 8); 3) la politización, factor que se refiere "[...] al grado en el cual los políticos están involucrados en realizar nominaciones partidistas a los puestos altos de la burocracia”. Dentro de los casos con menos politización de la burocracia, encontramos el caso británico, sin embargo, la gran mayoría de casos presentan niveles considerables de politización, como Bélgica, Austria, Italia, donde según Page y Wright (1999: 9) “(...) el pertenecer a un partido político o al menos tener conexiones personales con algún jefe de partido puede ser una condición importante para el reclutamiento y la promoción"; y iv) la subordinación política, que se refiere a "(...) aquellas instituciones donde el ministro busca ejercer control sobre las actividades de la burocracia, incluyendo el desarrollo de la política pública al interior del ejecutivo".

5 Un ejemplo por excelencia del primer patrón de reclutamiento mencionado son los casos de Francia e Inglaterra, donde la formación en la École Nationale D' Administration (ENA) u otras instituciones en el caso de Francia, o de Oxford o Cambridge en el caso Inglés, son determinantes para la ocupación de altas posiciones en la burocracia; 
Con respecto a los roles políticos de la burocracia, Page y Jenkins (2005) definen su influencia en dos principales tipos de trabajo: producción de política pública y mantención de política pública. Por producción de política pública, definen aquel trabajo que está "asociado a la elaboración de documentos, borradores o declaraciones" (Page y Jenkins, 2005: 60), siendo el más importante desde el punto de vista de la influencia política el relacionado con la elaboración de la legislación secundaria (reglamentos, decretos, resoluciones). Con este tipo de trabajo los burócratas de alto nivel influencian en el proceso de formulación de política ya que este no solamente implica la elaboración misma de los documentos, sino también el tratar con diferentes grupos de interés u organizaciones interesadas en ciertas políticas públicas, intercambiando posturas a través de relaciones de colaboración (Page y Jenkins, 2005: 66). Por otro lado, el trabajo de mantención de política pública, está relacionado con la realización de "(...) recomendaciones día a día sobre decisiones relacionadas con un esquema particular o conjunto de instituciones sobre cómo pueden ser manejadas" (Page y Jenkins, 2005: 60). Dentro de este trabajo, Page (2012) menciona el seguimiento en el avance de la implementación de las políticas públicas.

En relación al rol político de los burócratas, Howlett y Walker (2012) identifican un tipo de experticia desarrollada por los burócratas distinta a la de los tecnócratas o tecnopolíticos: la experticia de política pública, definida como el conocimiento de un “(...) rango de políticas e instrumentos, presentes y pasados, propuestos y promulgados, que rigen en un área de política en particular, conociendo cómo estos operan"; el "conocimiento de procesos complejos que deben ser seguidos para asegurar que una propuesta pueda ser puesta en efecto" (Howlett y Walker 2012: 259). Para Colebatch (2006: 315), “(...) a menudo es un conocimiento tácito, el cual no puede ser explícitamente codificado, sino que descansa mucho en prácticas personales o institucionales implícitas". La experticia de política pública también tendría relación con la capacidad de comprender el entorno político en el cual se da la discusión de política pública. En esta línea, Hoppe y Jeliazkova (2006: 42) destacan de los burócratas el conocimiento del proceso político y las destrezas para desenvolverse en estos contextos e instituciones específicas. La experticia también abarcaría la posesión de habilidades para facilitar la interrelación entre distintos actores “(...) para adquirir e intercambiar información, comunicar ideas y persuadir e influenciar individuos y organizaciones para apoyar un curso de acción" (Mintrom 2003: 102). Adams, Colebatch y Walker (2015: 103) evidencian que el trabajo de estos no está relacionado con "(...) un ejercicio abstracto en modelar problemas y demostrar soluciones sino que está más relacionado con construir programas de acción y negociar las partes jugadas por otros participantes". En específico nos referimos a 
habilidades de negociación, comunicación, persuasión, entre otras.

\section{METODOLOGÍA}

El propósito del presente estudio es analizar a los burócratas de nivel medioalto en Chile como élite, en relación a las dos perspectivas planteadas por Page y Wright (1999): status político en cada una de sus dimensiones (patrón de reclutamiento, estructura de carrera, politización y subordinación política) y roles políticos, analizando estos roles en función de los diferentes tipos de trabajo (producción de política pública, mantención de política pública y mantención de política pública) y la experticia de política pública.

La presente investigación es un estudio de caso de tipo cualitativo, empírico y no experimental. El caso de análisis seleccionado es la política de modernización de la gestión pública Yin (1994) plantea que el estudio de caso es una estrategia útil especialmente cuando la pregunta que orienta la investigación apunta al cómo, o cuando no se tiene mayor control sobres los eventos analizados. Junto con lo anterior, el estudio de caso permite buscar la mejor explicación dentro de una serie de eventos. El análisis de la política de modernización de la gestión pública ${ }^{6}$ cubrirá el período 1990-2014, el cual abarca cinco períodos presidenciales, de los cuales cuatro pertenecen a la coalición de partidos de centro izquierda denominada Concertación de Partidos por la Democracia (1990-2010) y uno a la coalición de centro derecha denominada Coalición por el Cambio (2010-2014).

Para el caso chileno se consideró como burócratas a aquellos actores que ocuparon cargos correspondientes a primer nivel jerárquico (directivos públicos), segundo y tercer nivel jerárquico (jefe de división o jefe de departamento). Estos actores se encontrarían en un nivel inmediatamente inferior de los cargos designados políticamente como los ministros o subsecretarios, de acuerdo al ordenamiento institucional chileno formal. En el caso chileno, desde el primer nivel jerárquico hacia abajo la función que les corresponde es la implementación de políticas públicas (Longo 2013), estando reservada la función política para los ministros y subsecretarios. Cabe señalar que, aunque estos actores puedan pertenecer a un partido político o red política, la función que han desempeñado o desempeñaron

6 A su vez, esta política ha estado conformada por cinco programas e iniciativas: el establecimiento del Sistema de Control de Gestión, el Sistema de Compras Públicas, la creación del Servicio Civil, el programa de Transparencia y Acceso a la Información Pública y el programa en busca de mejorar la efectividad en la gestión pública o ChileGestiona. 
en el contexto de la política de modernización de la gestión pública ha estado relacionada con un aspecto burocrático más que político.

La unidad de análisis son los burócratas de nivel medio-alto. Los datos recabados provienen de cuatro fuentes: entrevistas, documentación oficial, bibliografía académica y notas de prensa ${ }^{7}$. En este sentido, en primer lugar, se entrevistó a 48 actores, políticos, directivos públicos, representantes de funcionarios públicos y académicos, quienes identificaron a los burócratas clave que participaron en la política de modernización de la gestión pública. Posteriormente, se entrevistó a 30 burócratas considerados clave, profundizando en sus trayectorias y mecanismos de influencia. La importancia de profundizar en el conocimiento de estos burócratas tiene relación porque gran una gran mayoría de los actores políticos entrevistados señalaron que en la formulación de la política de modernización de la gestión pública existió una mínima presencia de los políticos en el diseño mismo, siendo más bien los burócratas quienes tuvieron una influencia gravitante. Los entrevistados fueron seleccionados de acuerdo al criterio de muestreo teórico y redundancia teórica, permitiendo descubrir categorías analíticas (Valles 2007). En específico, para el análisis de los roles políticos ejercidos por los burócratas, las entrevistas fueron codificadas mediante el uso del software de análisis de datos cualitativos ATLAS Ti, en función de la clasificación de las siguientes variables propuestas por Page y Jenkins (2005).

\section{RESULTADOS}

A continuación, y de modo introductorio, se entregarán los principales antecedentes de la política de modernización de la gestión pública en Chile entre 1990 y 2010, para posteriormente exponer el análisis de los resultados obtenidos en relación a las características y mecanismos de influencia de los burócratas que participaron de esta política pública, tanto a través de un análisis estadístico descriptivo que evidencia las principales características de este grupo, así como del análisis de contenido de las entrevistas realizadas (status políticos y roles políticos)

\section{LA POLÍTICA DE MOdERNIZACión dE LA GESTIÓN PÚBliCA EN ChILE}

Olavarría (2010) analiza la política de modernización de la gestión pública desde la óptica del proceso político, señalando como uno de sus rasgos característicos durante los gobiernos de la Concertación fue el escaso interés

7 Esta información fue recogida en el marco del Proyecto Fondecyt 1120546, a cargo de Mauricio Olavarría Gambi. 
de los políticos por la modernización de la gestión pública. A comienzos de la década de 1990 la modernización de la gestión pública sería más bien el producto del impulso de algunos burócratas de nivel medio al intentar modernizar los propios servicios en donde ellos se desempeńaban ${ }^{8}$.

Olavarría (2010) señala como hito importante el gobierno del Presidente Frei Ruiz-Tagle (1994-2000), en el cual se formaliza la política de modernización de la gestión pública, ya que instala el tema de la modernización de la gestión pública en la agenda pública. Frei Ruiz-Tagle crea el Comité Interministerial de Modernización de la Gestión Pública con el objetivo de contar con una agencia que gestionara la política, bajo la Secretaría General de la Presidencia (SEGPRES), y con un fuerte influjo de la Dirección de Presupuestos (DIPRES) a través de la Subdirección de Racionalización Administrativa. No obstante lo anterior, tuvo un diseño en etapas germinales, y "se basó en reformas de la gestión de servicios públicos determinados, que se iban implementando de acuerdo a las posibilidades que se tenían" (Olavarría 2010: 66).

La política de modernización de la gestión pública tampoco fue una prioridad en la agenda de gobierno del Presidente Lagos (2000-2006), por lo que pierde prioridad política siendo dirigido por el Proyecto de Reforma y Modernización del Estado (PRYME) (Olavarría 2010: 71). No obstante, con la aparición de los casos de corrupción MOP-GATE, MOP-CIADE, MOP-IDECON y CORFO-INVERLINK ${ }^{9}$, se genera una ventana de oportunidad para que la modernización de la gestión pública se instale como un tema relevante de la agenda. Producto de lo anterior, surgen los denominados Acuerdos Político-Legislativos para la Modernización del Estado, la Transparencia y la Promoción del Crecimiento, siendo la creación del Servicio Civil y el establecimiento del Sistema de Alta Dirección Pública (SADP) los resultados más importantes en materia de modernización en este período.

Durante el primer período de gobierno de la presidenta Michelle Bachelet (2006-2010) la modernización de la gestión pública tampoco fue un tema relevante, por lo que no es considerado como prioridad en la agenda de gobierno. Sin embargo, la aparición del caso de corrupción denominado

8 Olavarría (2010) señala que los servicios públicos en los que comenzó esta innovación son el Servicio de Impuestos Internos (SII), Instituto de Normalización Previsional (INP), Servicio de Registro Civil e Identificación y el Fondo Nacional de Salud (FONASA).

9 Para conocer en mayor detalle cada uno de estos casos, ver Olavarría (2010: 72-73). 
Chiledeportes ${ }^{10}$, genera una ventana de oportunidad y la modernización encuentra un espacio en la discusión pública. Producto de lo anterior se crea en el ańo 2006 la Comisión por la Probidad y la Gestión Pública, la que plantea la necesidad de establecer una política de transparencia y acceso a la información pública, que concluye con la creación del Consejo para la Transparencia con el objetivo de gestionar esta política.

\section{Status político}

\section{Patrón de Reclutamiento}

$\mathrm{Al}$ analizar el patrón de reclutamiento de los burócratas entrevistados, y en relación con la universidad de origen, se observa que más del $70 \%$ proviene de la Pontificia Universidad Católica de Chile (40\%) o la Universidad de Chile $(33,3 \%)$, ambas consideradas las mejores universidades del país y donde se forma parte importante de la élite intelectual y profesional del país (Neira 2004).

\section{Tabla 1: Universidad de origen de los burócratas}

\begin{tabular}{cccc} 
Universidad & Frecuencia & Porcentaje & $\begin{array}{c}\text { Porcentaje } \\
\text { acumulado }\end{array}$ \\
\hline Universidad de Chile & 10 & 33,3 & 33,3 \\
Pontificia Universidad Católica de Chile & 12 & 40,0 & 73,3 \\
Universidad de Santiago de Chile & 3 & 10,0 & 83,3 \\
Otra universidad del Consejo de Rectores & 2 & 6,7 & 90 \\
(Chile) & 3 & 10,0 & 100 \\
Otra universidad & 30 & 100 & 100
\end{tabular}

Nota: El Consejo de Rectores agrupa a 25 universidades públicas y otras tradicionales del país (existentes antes de la reforma de 1981, que permitió la creación de universidades privadas).

Fuente: Elaboración propia.

Por otro lado, con respecto a la especialización necesaria para ser parte del sector público en Chile observamos que no es condición exclusiva la formación en alguna institución educacional en particular (como el caso de la ENA en Francia), ni tampoco haber cursado estudios de pregrado relacionados con gestión o política pública. La mayoría de los entrevistados

10 Básicamente, este caso se desencadena por el descubrimiento que al interior de ChileDeportes, servicio público destinado a promover proyectos y actividades deportivas, se realizaron traspasos de dineros para el financiamiento de campañas políticas. 
tiene un título de grado de economista (33,3\%), de abogado (20\%) o ingeniero (20\%). La especialización de estos funcionarios se evidencia en la realización de estudios de postgrado, en específico de maestría (más del $83 \%$ posee un grado de magíster). También llama la atención que de los entrevistados solo uno posee un grado de doctor. En otras palabras, la especialización es más profesional que académica.

Donde mayormente podemos apreciar la especialización de los burócratas en el caso analizado tiene relación con el área de la maestría cursada. Complementando la información entregada por la tabla anterior, podemos observar que el área mayoritaria de especialización de postgrado es en gestión pública, ciencia política o políticas públicas (36,7\%), estando en segundo lugar de importancia la especialización en el área económica. Este carácter más práctico que académico de los postgrados de los burócratas lo diferenciaría de los tecnócratas y los tecnopolíticos.

\section{Tabla 2: Área de postgrado de burócratas} Frecuencia

\section{Porcentaje}

20,0

16,7

36,7

10,0

3,3

13,3

100
Porcentaje acumulado

20,0

36,7

73,3

83,3

86,7

100

100

Total

30

Fuente: Elaboración propia.

En síntesis, podemos señalar que existe en los burócratas entrevistados un factor de especialización en conocimientos prácticos más que un conocimiento generalista como lo es en el caso inglés o francés. Sin embargo, por otro lado en relación con el origen de la formación de pregrado, el caso chileno sería más similar al inglés en relación a la formación en instituciones de élite, pues la gran mayoría de los burócratas en altas posiciones han egresado de las dos instituciones más prestigiosas.

\section{Estructura de carrera}

Con respecto a la estructura de carrera de los burócratas analizados se cumple lo señalado por Page y Wright (1999) en relación a que, en los casos donde existe una mayor especialización de la élite, estos permanecen por 
mayor tiempo en sus cargos. De los burócratas entrevistados, el promedio de años que llevan ejerciendo en el sector público es superior a los 9 años

Otro dato que aporta antecedentes importantes es la cantidad de cargos que han desempeñado en el sector público los burócratas entrevistados. En promedio, han tenido aproximadamente 2,5 cargos durante su ejercicio. Llama la atención que los burócratas entrevistados del gobierno de Sebastián Piñera no habían desempeñado cargos anteriores en la burocracia estatal, proviniendo principalmente del sector privado o la academia, a diferencia de los burócratas que participaron de los gobiernos de la Concertación, quienes en, promedio, tuvieron aproximadamente 3 cargos en el sector público.

Para analizar la trayectoria de los entrevistados, se ha construido un patrón de permanencia o ascenso de los burócratas durante su permanencia en el sector público. En este sentido llama la atención la baja movilidad a cargos superiores de los burócratas. Más del 73\% de los entrevistados, aunque pudieron rotar en diferentes cargos en el sector público, permanecieron en el mismo nivel jerárquico de origen. Se observa una casi nula progresión de un segundo nivel jerárquico a un primer nivel jerárquico $(16,7 \%$ de los entrevistados). De igual forma, es escasa la movilidad de burócratas que se encuentran en la burocracia tradicional (en el sentido formal institucional) a cargos de confianza política tipificados en la ley (como subsecretarios, ministros, intendentes, entre otros) (solo el 6,7\% de los entrevistados).

\section{Tabla 3: Análisis de trayectorias de burócratas}

\begin{tabular}{lccc} 
& Frecuencia & Porcentaje & $\begin{array}{c}\text { Porcentaje } \\
\text { acumulado }\end{array}$ \\
\hline $\begin{array}{l}\text { Se mantiene en el } \\
\text { mismo nivel }\end{array}$ & 22 & 73,3 & 73,3 \\
$\begin{array}{l}\text { Asciende de jefe a } \\
\text { directivo público }\end{array}$ & 5 & 16,7 & 90,0 \\
$\begin{array}{l}\text { Asciende de } \\
\text { directivo a cargo }\end{array}$ & 2 & 6,7 & 96,7 \\
político \\
$\begin{array}{l}\text { Desciende de cargo } \\
\text { político a directivo } \\
\text { público } \\
\text { Total }\end{array}$ & 1 & & 100 \\
\end{tabular}




\section{Politización}

Como mencionamos en la introducción, diferentes investigaciones (Ferraro 2008, Rehren 2002, 2004), evidencian el carácter altamente politizado de la burocracia chilena, desde un punto de vista negativo, asociado principalmente a prácticas clientelares en la ocupación de puestos en la burocracia. A la analizar la información entregada por los burócratas entrevistados, podemos observar una cantidad considerable de estos actores que milita en partidos políticos (33,3\%). Sin embargo, una de las dificultades metodológicas de esta investigación tiene que ver con la falta de información sobre militancia política de los burócratas entrevistados, razón por la cual una gran cantidad de ellos aparecen rotulados como "sin información”.

Con el objetivo de complementar la información anteriormente expuesta, analizamos la información con respecto a que coalición de partidos gobernaba mientras los burócratas ejercían sus cargos. En este sentido, se evidencia que la gran mayoría de los entrevistados $(66,7 \%)$ ejerció su cargo solamente bajo los gobiernos de una coalición gubernamental. De los diez entrevistados que ejercieron entre distintas coaliciones políticas, es necesario precisar que cinco de ellos (16,7\%) pertenecían al Consejo para la Transparencia, corporación autónoma de derecho público, que no está tan inmersa a los vaivenes políticos como otras reparticiones de gobierno. $\mathrm{Si}$ extraemos estos cinco casos, podemos constatar que solo el $16,4 \%$ de los entrevistados ejerció su cargo durante las dos coaliciones políticas. La anterior nos sirve como una variable proxy para explicar el alto nivel de politización de los cargos en el sector público en los niveles medio-alto de la burocracia, que están sujetos a la permanencia de determinada coalición en el gobierno. Esto ha sido evidenciado ya por diferentes investigaciones relacionadas con la rotación de altos directivos públicos seleccionados por el Sistema de Alta Dirección Pública en función del cambio de gobierno (Costa y Waissbluth 2007, Olavarría y Dockendörff 2016, GonzálezBustamante 2016)

\section{Subordinación política}

Analizando la subordinación política en el caso de la política de modernización de la gestión pública se destaca que los entrevistados evidencian el desinterés de los políticos por la formulación de la política misma, salvo cuando aparecen casos de corrupción de notoriedad pública. Un directivo público entrevistado señala que "en el año 90, nadie de los que estaban en el gobierno estaban pensando en eso (la modernización de la gestión pública)”. En síntesis, podemos señalar que en este caso existe 
una menor subordinación política de los burócratas que en el caso de una política donde los políticos estén activamente interesados.

\section{Roles POLÍTICOS}

A continuación se analiza el trabajo realizado por los burócratas en la política de modernización de la gestión pública de acuerdo al tipo de trabajo realizado por ellos, así como por la experticia de política pública (Page y Jenkins 2005).

\section{Tipo de trabajo \\ Producción de Política Pública}

La gran mayoría de los burócratas entrevistados participaron en la elaboración de informes o reportes donde tuvieron una parte activa en plantear diferentes propuestas de política pública. Cabe destacar que gran parte de las acciones en torno a la modernización de la gestión pública fue formalmente establecida mediante la figura de decretos o instructivos presidenciales, en específico durante la administración del presidente Frei Ruiz-Tagle. En los gobiernos de Lagos y Bachelet, producto de los casos de corrupción, se generan cuerpos legales que dan vida al Sistema de Alta Dirección Pública, al Sistema de Compras Públicas, y la Ley de Acceso a la Información y Transparencia). Los burócratas de nivel medio-alto en estos períodos tuvieron una influencia en la construcción de estas mismas leyes, así como en la creación de los reglamentos para operacionalizarlas ${ }^{11}$. En relación con el establecimiento del Programa de Control de Gestión en el período de Frei Ruiz-Tagle, y abordando las estrategias para su formulación, un burócrata de segundo nivel jerárquico señaló: "nosotros usamos todo el abanico, o sea nosotros usamos proyectos de Ley, dentro de los proyectos de ley pedimos Decretos de Fuerza de Ley, y usamos harto el instructivo presidencial".

Dentro de este tipo de trabajo destaca la relación de los burócratas con los diferentes actores involucrados en la política. De esta forma son capaces de visualizar de qué forma la política beneficia o perjudica a los diferentes actores involucrados, y generar estrategias para cada uno de estos grupos.

11 Como señala una asesora política: "la mayor parte de los instrumentos desde el 90 al 97 son instrumentos que no tienen carácter de ley, que son reglamentos o decretos, que son figuras más administrativas, instructivos por ejemplo, para modernizar el sistema de capacitación se lanza un instructivo presidencial [...] todo se maneja a nivel de decretos e instructivos, que son figuras menores desde el punto de vista legal, pero que también son muy efectivas". 
En este sentido un burócrata señala: "creo que hubo un esfuerzo en llamar no solamente a los jefes de servicios, sino que también a todo el personal, a los mismos sindicatos, a la ANEF [Asociación Nacional de Empleados Fiscales], a participar en este dialogo y eso se hizo en forma constante".

\section{Mantención de politica pública}

El seguimiento del cumplimiento de metas aparece casi en la mayoría de los casos de burócratas asociados al Programa de Control de Gestión y desde la Dirección de Presupuestos, realizando un seguimiento del estado de las metas comprometidas anualmente por el gobierno. Esto se debería principalmente a que en esta etapa se establecieron los Programas de Mejoramiento de Gestión (PMG), un mecanismo de incentivo monetario asociado al cumplimiento de metas (Pliscoff 2005). El seguimiento del cumplimiento de estas metas la realizan un tipo de burócratas denominados sectorialistas, asociados a la Dirección de Presupuesto (Arenas y Berner 2010). Los burócratas que participan en el seguimiento de objetivos influyen en el proceso de formulación de política, ya que con la evaluación que desarrollan influyen en la asignación presupuestaria de ciertas políticas (creación de incentivos). En este sentido el seguimiento está más relacionado con el cumplimiento de objetivos políticos. Un burócrata entrevistado señaló: "Entonces, lo que buscamos fue alinear los indicadores de los servicios públicos que estaban en la discusión del presupuesto anual, los indicadores, que estuvieran correctamente alineados con los objetivos generales de cada ministerio". Otro burócrata que trabajo durante el período del Presidente Piñera señaló: "Y así trabajamos en la unidad presidencial de gestión y cumplimiento estableciendo objetivos y resultados en materia educativa, en mejora de los puntajes de acceso a las carreras de pedagogías, en materia de seguridad ciudadana, en materia de pobreza”.

\section{EXPERTICIA DE POLÍTICA PÚBLICA}

La experticia de política pública se ve evidenciada en la habilidad de los burócratas para negociar y persuadir a los actores de poder en relación con los alcances de las políticas que están diseñando y/o implementando. La experticia de política pública estaría caracterizada también por el desarrollo de importantes competencias transversales o habilidades blandas. Un político señala en relación con los profesionales que ocupan cargos de dirección pública en el Estado que "la gente que viene con vocación y que tienen habilidades blandas muy desarrolladas, pueden sobrevivir estupendo y tener formas que nadie había dimensionado". Otra de las características transversales de los burócratas analizados es la facilidad de acceso a las 
autoridades políticas, que les permitiría tener el espacio propicio para poder participar en la definición de las políticas públicas. Un directivo público asociado a la Política de Transparencia comprende la necesidad de tener vías de comunicación directa con el Poder Ejecutivo: "lo que se produjo ahí un llamado telefónico, a la presidenta, CON quien históricamente ya habíamos iniciado relaciones, la conocíamos como sociedad civil básicamente dos años antes, habíamos conocido su experiencia”.

\section{DISCUSIÓN}

¿Qué nos dice la evidencia analizada con respecto a los burócratas como elite gubernamental? El principal hallazgo de esta investigación es la identificación de los burócratas ejerciendo influencia política en la formulación de políticas, exhibiendo un capital diferente al de los tecnócratas, tecnopolíticos y políticos profesionales. Las características más frecuentes de este burócrata de nivel medio en el caso de la política de modernización de la gestión pública son: haber realizado sus estudios de pregrado en la Pontificia Universidad Católica de Chile o la Universidad de Chile, poseer títulos profesionales de economista, ingeniero o abogado, poseer una maestría en gestión o políticas públicas o administración, tener una experiencia de más de nueve ańos en cargos directivos en la burocracia estatal, sin ascender a cargos de confianza política (ministro, subsecretario, intendente, por ejemplo).

Junto con lo anterior, la evidencia demuestra que la gran mayoría de estos burócratas no pertenecen formalmente a un partido político, no obstante en la mayoría de los casos es posible visualizar la pertenencia de estos a redes cercanas al mundo político. La cercanía de los burócratas con los actores políticos podría explicarse por la práctica política informal en Chile denominada cuoteo, a través de la que se designan cargos en la administración pública de acuerdo a una distribución de cupos entre los partidos políticos de la coalición ganadora (Dávila 2009). Si bien no podemos extrapolar estos hallazgos a toda la administración pública, investigaciones como las de Olavarría (2015), Olavarría y Dockendorff, (2016), Grindle, (2012), Rehren (2010), nos hacen presumir que esta es una característica común de los burócratas ubicados en los mandos medios en el caso chileno. Estos hallazgos confirmarían que en el caso de la política de modernización de la gestión pública, los burócratas de mando medio jugarían un rol cuasipolítico, posicionándolos como un actor de poder influyente en el proceso de formulación de política pública, y no tan solo como un mero implementador o ejecutor de política pública.

En el caso de la política de modernización de la gestión pública es 
posible observar diferencias importantes entre el rol ejercido por los tecnócratas, los tecnopolíticos y los burócratas. En el caso analizado en este estudio apreciamos que los burócratas no influenciarían en función de la experticia técnica reflejado en la posesión de grados doctorales en prestigiosas universidades internacionales, sino más bien por la considerable trayectoria en el sector público en cargos burocráticos similares, la posición jerárquica de estos en los tres primeros niveles jerárquicos de la burocracia chilena y cierta experticia técnica evidenciada en la posesión de grados de magíster, principalmente en el área de la gestión pública. La sumatoria de todos estos factores permitiría el desarrollo de una experticia práctica en los burócratas, siendo capaces de comprender el entorno político en el que se desenvuelven y desarrollar las destrezas necesarias para desenvolverse en ese contexto, como persuasión, negociación, comunicación, entre otra). Esta conjunción de elementos, puede decantar en lo que Bourdieu (2005) denomina capital burocrático, asociado tanto a la posición que se ocupa en la jerarquía como a la antigüedad en la organización. Además, este capital también estaría relacionado con la posesión de conocimiento sobre cómo operan distintos procesos al interior del Estado, adquiridos principalmente por la experiencia personal. En la misma línea de Bourdieu (2005), Cantu (2009: 13), también se refiere al capital burocrático como un "recurso fruto de distintas trayectorias (al interior del Estado)". Por otro lado, Perdomo y García (2010: 166) entienden el capital burocrático como "el poder que otorga ocupar un cargo en la jerarquía organizacional (del Estado)". Por lo tanto, la evidencia presentada en este caso nos lleva a concluir que los burócratas poseen un capital diferente de los tecnócratas y tecnopolíticos: el capital burocrático.

\section{Tabla 4: Diferencias entre actores que influencian el proceso de formulación de política pública}

\section{Burócrata Tecnócrata Tecnopolítico}

Posición jerárquica Medio-Alto Alto Alto

Fuente de influencia en el proceso de formulación de política

Experticia de Experticia Cien-

Política Pública tífica

Tipo de capital Burocrático
Experticia Científica/ Influencia política (capital político)

Tecnocrático/ Político

Fuente: Elaboración propia.

Llama la atención los hallazgos de esta investigación en relación a la diferencia de capital entre los burócratas de los gobiernos de la 
Concertación versus los burócratas de la Coalición por el Cambio, evidenciada principalmente en la trayectoria en el sector público así como la experiencia en cargos directivos en el sector público previamente. De acuerdo a lo señalado por Silva (2010), el gobierno de Piñera privilegió llenar los cargos en la burocracia estatal por tecnócratas en el sentido estricto de la definición (doctorados en economía en universidades de prestigio, sin militancia política y escasas redes políticas, cierta apatía por el juego político, y con cargos previos en altas posiciones en el sector privado, principalmente en el mundo del retail). Esta orientación en la selección de los altos puestos en la burocracia tendría relación con la búsqueda de un sello en la gestión de excelencia, lo que provocó la remoción de una gran cantidad de funcionarios que ocupaban sus cargos desde los gobiernos de la Concertación, tanto desde el nivel directivo como en cargos medios (González-Bustamante et al. 2016). Por consiguiente, podemos inferir que en el caso analizado existe una mayor acumulación de capital burocrático en los burócratas del período de la Concertación que los pertenecientes a la Coalición por el Cambio.

El predominio de estas características en la burocracia es la que, según Silva (2010) determinó un constante conflicto entre estos tecnócratas y los partidos de gobierno. Pero no tan solo con los partidos políticos sino también con distintos movimientos sociales ${ }^{12}$, especialmente el movimiento estudiantil de 2011, provocando complejidades en la gobernabilidad. En específico se cuestionó la capacidad del gobierno de Piñera en negociar con distintos grupos de interés, provocando constantes fisuras en la gobernabilidad durante su período de gobierno. Como mencionamos en la revisión teórica, una gran parte de esta actividad de negociación y persuasión con los diferentes grupos de interés es una actividad de los cargos medios-altos de la burocracia (Wu et al. 2015). Por consiguiente, los problemas de gobernabilidad durante el gobierno de Pińera podrían explicarse, en parte, por el escaso capital burocrático de los burócratas de nivel medio-alto que formaron parte de su gobierno. Si bien, quienes ocuparon cargos en los niveles medios-altos de la burocracia en el gobierno de Piñera tenían una alta experticia científica, carecían de la Experticia de Política Pública o capital burocrático. En relación con estos hallazgos toma fuerza la propuesta de Grindle (2012) al señalar ciertos efectos positivos del patronazgo como mecanismo de reclutamiento de los burócratas, además

12 Dentro de los movimientos sociales, quizás el de mayor importancia mediática fueron las movilizaciones estudiantiles de 2011 con un foco en la eliminación del lucro en la educación, el fortalecimiento de la educación pública y una mejora en la calidad de la educación en general. Otros movimientos sociales fueron el Movimiento por Hidroaysen. 
de determinar la estructura de carrera, ya que estos podrían estimular el desarrollo de la experticia de política pública y una mayor acumulación de capital burocrático afectando la gobernabilidad de un país. En el caso chileno, esto podría explicar la diferencia de capital burocrático entre los burócratas que pertenecieron a los gobiernos de la Concertación frente aquellos pertenecientes al de la Coalición por el Cambio.

Este estudio tuvo el propósito de evidenciar empíricamente cómo los burócratas de nivel medio influencian en el proceso de formulación de política pública en el caso de la política de modernización de la gestión pública en Chile. El aporte de esta investigación al debate teórico subyace en ser uno de los primeros estudios empíricos que analizan la influencia política de los burócratas de nivel medio en la formulación de política pública en Chile, describiendolos como actores distintos de los tecnócratas y los tecnopolíticos, especialmente con respecto al capital burocrático como elemento diferenciador de los burócratas. Junto con lo anterior, este es uno de los pocos trabajos en Chile que se centra en analizar tanto el status político como los roles políticos que ejercen los burócratas. Futuras investigaciones podrían cmedir el peso de los diferentes elementos analizados en la acumulación de capital burócratico en los burócratas de nivel medio, evaluando cual de ellos es más gravitante en el caso chileno. 


\section{REFERENCIAS}

Aberbach, J., Putnam, R. y Rockman, B. (1981). Bureaucrats and Politicians in Western Democracies. Cambridge: Harvard University Press.

Adams, D., Colebatch, H. y Walker, C. (2015). Learning about Learning: Discovering the Work of Policy. Australian Journal of Public Administration, 74 (2), 101-111.

Allison, G. (1971). Essence of Decision: Explaining the Cuban Missile Crisis. Little: Brown and Company.

Arenas, A. y Berner, H. (2010). Presupuesto por Resultados y la Consolidación del Sistema de Evaluación y Control de Gestión del Gobierno Central. Santiago: Dirección de Presupuestos.

Baylis, T (1974). The Technical Intelligentsia and the East German Elite. Berkeley: University of California Press.

Bourdieu, P. (2005). The Social Structures of the Economy. Malden: Polity.

Cantu, R. (2009). Uma Abordagem Da Burocracia Economica como Espaco Social. Ponencia presentada en el I Seminário Nacional Sociología \& Política, Universidade Federal do Paraná, Paraná, Brasil.

Carpenter, D. (2001). The Forging of Bureaucratic Autonomy: Reputations, Networks, and Policy Innovation in Executive Agencies, 1862-1928. Princenton: Princeton University Press.

Centeno, M. (1993). The New Leviathan: The Dynamics and Limits of Technocracy. Theory and Society, 22 (3), 307-335.

Centeno, M. y Maxfield, S. (1992). The Marriage of Finance and Order: Changes in the Mexican Political Elite. Journal of Latin American Studies, 24 (1), 57-85.

Cheng, L. y White, L. (1990). Elite Transformation and Modern Change in Mainland China and Taiwan: Empirical Data and the Theory of Technocracy. The China Quarterly, 121, 1-35.

Colebatch, H. (Ed.). (2006). What Work Makes Policy? Policy Science, 39 (4), 309-321. 
Colebatch, H, Hoppe, R. y Noordegraaf, M. (2010). Working for Policy. Amsterdam: Amsterdan University Press.

Collier, S. (1979). The New Authoritarianism in Latin America. Princeton: Princeton University Press.

Costa, R. y Waissbluth, M. (2007). Tres Años del Sistema de Alta Dirección Pública en Chile: Balances y Perspectovas. Santiago: Departamento Ingeniería Industrial, Universidad de Chile.

Dahlström, C. (2012). Politics and Administration. En Peters, B. G. y Pierre, J. (Ed.), The SAGE Handbook of Public Administration. Londres: SAGE.

Davila, M. (2009). Governing Together: The Concertation Administrations in Chile (1900-2009). Tesis (PhD in Political Science), The University of North Carolina at Chapel Hill, Estados Unidos.

. (2011). Tecnocracia y Política en el Chile Posautoritario (1990-2010). En Joignant, A. y Güell, P. (Ed.), Notables, Tecnócratas y Mandarines: Elementos de Sociología de las Elites en Chile (1990-2010). Santiago: Ediciones Universidad Diego Portales.

Delamaza, Gonzalo. (2011). Economistas en la Élite: Entre Tecnopolítica y Tecnociencia. En Joignant, A. y Güell, P. (Ed.), Notables, Tecnócratas y Mandarines: Elementos de Sociología de las Elites en Chile (1990-2010). Santiago: Ediciones Universidad Diego Portales.

(2013). De la Elite Civil a la Elite Política. Reproduccion del Poder en Contextos de Democratización. Polis, 12 (36), 67-100.

Domínguez, J. (1997). Technopols: Freeing Politics and Markets in Latin America in the 1990s. College Park: Pennsylvania State University Press

Easton, D. (1965). A Systems Analysis of Political Life. Nueva York: Wiley.

Ferraro, A. (2008). Friends in High Places: Congressional Influence on the Bureaucracy in Chile. Latin America Politics and Society, 50 (2), 101-129.

Góngora, M. (1981). Ensayo Histórico sobre la Noción de Estado en Chile en los Siglos XIX y XX. Santiago: Editores La Ciudad. 
González-Bustamante, B., Olivares, A., Abarca, P. y Molina, E. (2016). Servicio Civil en Chile: Análisis de los Directivos de Primer Nivel Jerárquico (2003-13). Revista Administração Pública, 50 (1), 59-80.

Goodnow, F. (1900). Politics and Administration: A Study in Government. Nueva York: MacMillan.

Grindle, M. (2012). Jobs for the Boys. Cambridge: Harvard University Press.

Günell, J. (1982). The Technocratic Image and the Theory of Technocracy. Technology Culture, 23 (3), 392-416.

Hoppe, R. y Jeliazkova, M. (2006). How Policymakers Define Their Jobs: A Netherlands Case Study. En Colebatch, H. (Ed.), The Work of Policy: An International Survey. Oxford:Lexington Books.

Howlett, M. y Walker, R. (2012). Public Managers in the Policy Process: More Evidence on the Missing Variable? Policy Studies Journal, 4 (2), 211-233.

Hunneus, C. (1998). Tecnócratas y Políticos en un Régimen Autoritario: Los "ODEPLAN Boys" y los "Gremialistas" en el Régimen de Pinochet. Ciencia Politica, XIX (2) 125-158.

Jáksic, I. (2013). Ideological Pragmatism and Nonpartisan Expertise in Nineteenth-Century Chile: Andrés Bello's Contribution to State and Nation Building. En Centeno, M. y Ferraro, A. (Ed.), State and Nation Making in Latin America and Spain: Republics of the Possible. Nueva York: Cambridge University Press.

Joignant, A. (2009). El Estudio de las Elites. Un Estado del Arte. Santiago: Universidad Diego Portales.

. (2011). The Politics of Technopols: Resources, Political Competence and Collective Leadership in Chile, 1990-2010. Journal of Latin American Studies, 43 (3), 517-546.

Joignant, A. y Güell, P. (Ed.). (2011). Notables, Tecnócratas y Mandarines: Elementos de Sociología de las Elites en Chile (1990-2010). Santiago: Ediciones Universidad Diego Portales. 
Lasswell, H. (1936). Politics: Who Gets What, When, How. Nueva York: Wittlesey House.

Longo, F. (2013). Diez años de Alta Dirección Pública en Chile. Ponencia presentada en el XVIII Congreso Internacional del CLAD sobre la Reforma del Estado y de la Administración Pública, 29 de octubre al $1^{\circ}$ de noviembre, Montevideo, Uruguay.

Mangset, M. (2015a). Contextually Bound Authoritative Knowledge: A Comparative Study of British, French and Norwegian Administrative Elite' Merit and Skills. En Van Zanten, A., Ball S. y Darchy-Koechlin, B. (Ed.), World Yearbook of Education: Elites, Privileges and Excellence: The National and Global Redefinition of Educational Advantage. Londres: Routledge.

. (2015). What Does it Mean To Be Part of Elite?: Comparing Norwegian, French and British Top Bureaucrats' Understandings of the Elite Concept When Applied to Themselves. Comparative Sociology, 14 (2), 274-299.

Mella, M. (2008). Los Intelectuales de los Centros Académicos Independientes y el Surgimiento de la Concertación. Revista de Historia Social y las Mentalidades: sociedad, Politica y Cultura, 1 (12), 83-121.

Merton, R. (1957). Social Theory and Social Structure. Glencoe: The Free Press.

Mintrom, M. (2003). People Skills for Policy Analyst. Washington: Georgetown University Press.

Mizuno, H. (2009). Science for the Empire. Scientific Nationalism in Modern Japan. Stanford: Stanford University Press.

Montecinos, V. y Markoff, J. (2009). Economists in the Americas. Cheltenham: Edward Eldgar.

Neira, H. (2004). Educación Universitaria en Chile: Una Visión Panorámica Centrada en los Alumnos. Estudios Pedagógicos, 30, 123133.

Noordegraaf, M. (2015). Public Management: Performance, Professionalism and Politics. Londres: Palgrave. 
O’Donnell, G. (1992). Delegative Democracy? Indiana: Kellogg Institute, University of Notre Dame.

Olavarría, M. (2010). ¿Cómo Se Formulan las Políticas Públicas en Chile? Tomo 1: La Modernización de la Gestión Pública. Santiago: Editorial Universitaria.

---. (2015). Agenda and Public Policy: Evidence from Chile. International Journal of Public Administration, 39 (2), 157-172.

Olavarría, M. y Dockendorff, A. (2016). Implementing Meritocracy in Senior Public Administration: The Dilemma for Chilean Politics. Public Organization Review, 16 (4). 561-582.

Osborne, D. y Gaebler, T. (1992). Reinventing Government: How the Entrepreneurial Spirit is Transforming the Public Sector. Reading: Addison-Wesley.

Overeem, P. (2005). The Value of the Dichotomy: Politics, Administration, and The Polítical Neutrality of Administrators. Administrative Theory \& Praxis, 25 (2), 311-329.

. (2006) In Defense of the Dichotomy: A Response to James H. Svara. Administrative Theory \& Praxis, 28 (1), 140-147.

Page, E. (2012). Policy Without Politicians: Bureaucratic Influence in Comparative Perspective. Oxford: Oxford University Press.

Page, E. y Jenkins, B. (2005). Policy Bureaucracy. Government with a Cast of Thousands. Londres: Oxford University Press.

Page, E. y Wright, V. (1999). Bureaucratic Elites in Western European States: A Comparative Analysis of Top Officials. Oxford: Oxford University Press.

Perdomo, A. y Martinez, P. (2010). Agentes y Campos Sociales en la Seguridad del Paciente de Tres Hospitales de Bogotá. Revista Gerencia Política Salud. 9 (10), 150-178.

Peters, B. G. (1995). The Politics of Bureaucracy. Nueva York: Longman Publisher. 
Putnam, R. (1977). Elite Transformations in Advanced Industrial Societies: An Empirical Assessment of the Theory of Technocracy. Comparative Political Studies, 10 (3), 383-412.

Pliscoff, C. (2005). Sistemas de Incentivos Monetarios y Reforma del Estado: Elementos para una Discusión Necesaria. Santiago: Instituto de Asuntos Públicos, Universidad de Chile.

Ramesh, M. (2008). Policy-Implementation Frame: A Revisit. South Asian Journal of Management, 15 (1), 42-63.

Rehren, A. (2002). Clientelismo Politico, Corrupción y Reforma del Estado en Chile. En S. Valdes, Reforma del Estado Vol II. Santiago: Centro de Estudios Públicos.

. (2004). Politics and Corruption, the Underside of Chilean Democracy. Harvard Review of Latin America, (spring), 1415.

- (2010). Clientelismo Político, Corrupción y Reforma del Estado en Chile. En Valdés, S. (Ed.), Reforma del Estado, Volumen II: Dirección Pública y Compras Públicas, Santiago: Centro de Estudios Públicos

Ribbhagen, C. (2013). Technocracy within Representative State Technocratic Reasoning and Justification among Bureaucrats and Politicians. Gotemburgo: University of Gothenburg.

Ridley, F. (1966). French Technocracy and Comparative Government. Political Studies, 14 (1), 344-52.

Rouban, L. (2012). Politicization of the Civil Service. En Peters, B. G. y Pierre, J. (Ed.), The SAGE Handbook of Public Administration . Londres: SAGE.

Rowney, D. (1989). Transition to Technocracy. The Structural Origins of Soviet Administrative State. Londres: Cornell University Press.

Salazar, G. (2006). Construcción de Estado en Chile (1800-1837): Democracia de los "Pueblos", Militarismo Ciudadano. Golpismo Oligárquico. Santiago: Editorial Sudamericana. 
Samuels, D. y Shugart, S. (2003). Presidentialism, Elections and Representation. Journal of Theoretical Politics, 15 (1), 33-60.

Savoie, D. (1999). Governing from the Centre: The Concentration of Power in Canadian Politics. Toronto: University of Toronto Press.

Seidman, H. (1970). Politics, Position, and Power: The Dynamics of Federal Organization. Oxford: Oxford University Press.

Shuggart, M. y Haggard, S. (2001). Institutions and Public Policy in Presidential Systems. Nueva York: Cambridge University Press.

Shuggart, M. y Mainwaring, S. (1997). Presidentialism and Democracy in Latin America: Rethinking the Terms of the Debate. Nueva York: Cambridge University Press.

Silva, P. (2008). In the Name of Reason: Technocrats and Politics in Chile. College Park: Pennsylvania: Penn State University Press. . (2010). En el Nombre de la Razón: Tecnócratas y Politica en Chile. Santiago: Ediciones Universidad Diego Portales.

Simon, H. (1965). Administrative Behaviour. Nueva York: Free Press.

Stabili, M. (2003). El Sentimiento Aristocrático. Elites Chilenas Frente al Espejo (1860-1960). Santiago: Editorial Andrés Bello/ Centro de Investigaciones Barros Arana.

Stuven, A. (2000). La Seducción de un Orden: Las elites y la Construcción de Chile en las Polémicas Culturales y Politicas del Siglo XIX. Santiago: Ediciones Universidad CatólIca de Chile.

Svara, J. (2006). Complexity in Political-Administrative Relations and the Limits of the Dichotomy Concept. Administrative Theory \& Praxis, 28 (1), 121-139.

Valles, M. (2007). Técnicas Cualitativas de Investigación Social: Reflexión Metodológica y Práctica Social. Madrid: Editorial Síntesis.

Vicuña, M. (1996). El París Americano. La Oligarquía Chilena como Actor Urbano en el Siglo XIX. . Santiago: Universidad Finis Terrae-Museo Histórico Nacional. 
Vicuña, M. (2001). La Belle Époque Chilena. Alta Sociedad y Mujeres de Élite. Santiago: Catalonia.

Waldo, D. (1952). Development of Theory of Democratic Administration. American Political Science Review, 46 (1), 81-103.

Weber, M. (2006). La Ciencia como Profesión, la Política como Vocación. Madrid: Espasa Libros.

Williamson, J. (1994). The Political Economy of Policy Reform. Londres: Institute for International Economics

Wilson, J. (1989). Bureaucracy: What Government Agencies Do and Why They Do It. Nueva York: The Perseus Books Group.

Wilson, W. (1887). The Study of Public Administration. Political Science Quarterly, 2 (2), 197-222.

Wonka, A. (2007). Technocratic and Independent? The Appointment of European Commisioners and its Policy Implications. Journal of European Public Policy, 14 (2), 169-189.

Wu, X, Ramesh, M., Howlett, M. y Fritzen, S. (2015). The Public Policy Primer: Managing the Policy Process. Londres: Routledge.

Yin, R. (1994). Case Study Research: Design and Methods, Beverly Hills: Sage. 